\title{
Backbending current-voltage characteristic for an annular Josephson junction in a magnetic field
}

\author{
Alexey V. Ustinov \\ Physikalisches Institut III, Universität Erlangen-Nürnberg, Erwin-Rommel-Strasse 1, D-91058 Erlangen, Germany \\ Boris A. Malomed \\ Department of Interdisciplinary Studies, Faculty of Engineering, Tel Aviv University, Tel Aviv 69978, Israel \\ Edward Goldobin \\ Institut für Schicht- und Ionentechnik, Forschungszentrum Jülich, D-52425 Jülich, Germany
}

(Received 28 January 1999)

\begin{abstract}
Excitation of the Josephson plasma radiation by a fluxon moving in an annular Josephson junction is studied experimentally, numerically, and using an analytical approach. An externally applied magnetic field $H$ forms a cosinelike potential relief for the fluxon in a ring-shaped junction. The motion of the fluxon in the junction leads to an emission of plasma waves, which give rise to a resonance at a certain fluxon velocity. The experimental data agree well with numerical simulations which indicate a locking of the fluxon to the radiation frequency. The peculiar feature indicated by both experiment and numerical simulations is the shape of the resonance in the current-voltage $(I-V)$ characteristic which shows a clear backbending, with a negative differential resistance. The analytical approach developed in this work is based on the perturbation theory for radiation emission generated by a kink in the perturbed sine-Gordon equation. To explain the observed effect, we introduce an addition to the perturbation theory, which proves to be crucial for explanation of the backbending $I$ - $V$ curves: We take into account the fact that the background radiation field, supported by a balance between emission from the moving kink and dissipative absorption, narrows the junction's plasma frequency gap. In the case when the emission has a resonant character, even a small change of the gap produces a strong reciprocal effect on the emission power. Following this idea, we develop a fully analytical self-consistent approximation that readily allows us to obtain the backbending $I-V$ curves.
\end{abstract}

[S0163-1829(99)15225-1]

\section{INTRODUCTION}

A fluxon in a long Josephson junction carrying a magnetic flux quantum $\Phi_{0}$ is a well-known example of a solitary wave described by the perturbed sine-Gordon model. The motion of a sine-Gordon soliton (kink) in a spatially periodic potential is a classic problem which has been studied in many theoretical papers, starting from Refs. 1 and 2 (see a review). It has been shown that the soliton radiates small-amplitude waves with the plasma dispersion relation. The radiation frequency depends on the soliton velocity and the period of the potential. $^{2,3}$ It has been also predicted ${ }^{4}$ that in a periodically modulated junction the generated radiation should lead to resonances which appear as additional constant-voltage steps on the current-voltage $(I-V)$ characteristics. These resonances have been observed in experiments ${ }^{5}$ using a long Josephson junction with an artificially fabricated lattice of inhomogeneities. Such a realization of the periodic potential appears to be rather straightforward but it does not allow one to control the amplitude and shape of the effective potential during the experiment.

Ring-shaped (annular) long Josephson junctions serve as the best experimental systems for studying fluxon dynamics. Due to the magnetic flux quantization in a superconducting ring, the number of fluxons initially trapped in an annular junction is conserved. The soliton dynamics can be studied here under periodic boundary conditions. While the fabrication of annular Josephson tunnel junctions is rather easy, trapping of fluxons in them still remains a difficult art. Using different trapping techniques, both single-fluxon ${ }^{6}$ and multifluxon ${ }^{7}$ experiments have been reported with homogeneous annular junctions.

In this paper we present experiments, numerical simulations, and analysis of the dynamics of a single fluxon trapped in an annular Josephson junction which is placed in an externally applied magnetic field $H$. The geometry is schematically shown in Fig. 1. Due to the interaction of the fluxon

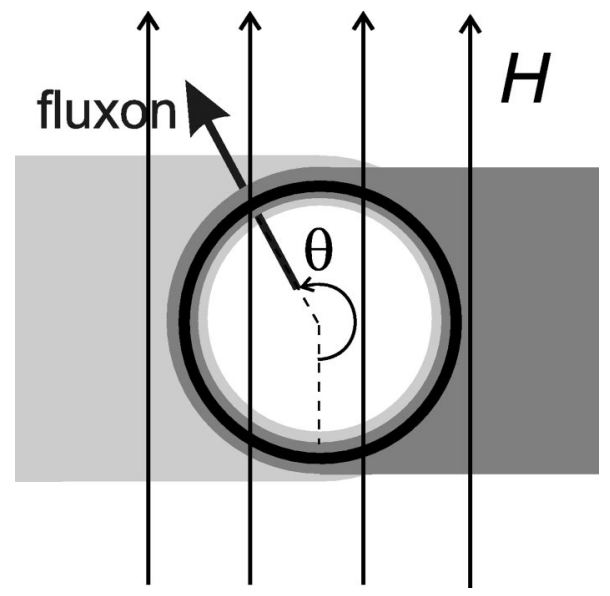

FIG. 1. Schematic view of an annular junction with a trapped fluxon; a magnetic field $H$ is applied in the plane of the tunnel barrier. 
with the radial field component, ${ }^{8}$ the fluxon feels a periodic potential $U(\theta) \sim H \cos \theta$. The minimum of the potential is located in the region of the ring where the fluxon's magnetic field is directed along the field. This problem has recently attracted considerable interest which has led to new experiments. ${ }^{9-12}$ Most of the work done thus far was focused on the fluxon pinning in the field-induced potential. Here we present a systematic study of the fluxon dynamics, strongly affected by nonlinear Josephson plasma wave radiation emission due to the fluxon motion. The linear (smallamplitude) case of radiation emission by a moving fluxon, leading to a new resonance observed in experiment, has been already reported in Ref. 10.

The theoretical model for this system was proposed by Gronbech-Jensen et al. ${ }^{8}$. The field gives rise to an additional term in the perturbed sine-Gordon equation which governs the fluxon motion:

$$
\varphi_{x x}-\varphi_{t t}-\sin \varphi=\alpha \varphi_{t}+\gamma+h \sin (q x),
$$

where $\varphi(x, t)$ is the superconducting phase difference between the electrodes of the junction, the spatial coordinate $x$ directed along the ring is normalized to the Josephson penetration depth $\lambda_{J}$, the time $t$ is normalized to the inverse plasma frequency $\omega_{0}^{-1}, \alpha$ is a dissipation coefficient due to the quasiparticle tunneling across the barrier, and $\gamma$ is the bias current density normalized to the critical current density $J_{c}$ of the junction. The parameter $q=2 \pi / l$, where $l$ $=\pi D / \lambda_{J}$ is the normalized circumference of the junction, with $D$ being the ring's diameter. The last term in Eq. (1) accounts for the coupling between the applied field and the flux density in the junction. The dimensionless amplitude $h$ $\sim H$ is normalized by a sample-specific geometrical factor. ${ }^{8,9}$ In case of one fluxon trapped in the ring, Eq. (1) is supplemented by the periodic boundary condition $\varphi(l)=\varphi(0)$ $+2 \pi$. At low velocities the fluxon's equation of motion that can be derived from Eq. (1), that of a driven pendulum in a lossy medium. ${ }^{13}$

\section{EXPERIMENTAL RESULTS}

Experiments have been performed on $\mathrm{Nb} / \mathrm{Al}-\mathrm{AlO}_{x} / \mathrm{Nb}$ Josephson junctions. Measurements were performed by applying the bias current $I$ from top to the bottom electrode of the junction and measuring the dc voltage generated due to the fluxon motion. The results presented below were obtained for a junction with mean diameter $D=132 \mu \mathrm{m}$ and ring width $W=10 \mu \mathrm{m}$. The normalized ring's circumference $l$ varied between 8.3 and 7.7.

Trapping of a magnetic flux in the junction was achieved while cooling the sample below the critical temperature $T_{c}^{\mathrm{Nb}}=9.2 \mathrm{~K}$ of niobium with a small bias current passing through the junction. Figure 2 shows the junction critical current dependence on magnetic field measured with no trapped fluxon (solid line) and with one fluxon trapped (points) during cooling down. This dependence is a very clear "fingerprint" of the junction state. With no fluxon $I_{c}(H)$ has the usual Fraunhofer-like form with maximum $I_{c}$ at zero field. In contrast, the junction state with a trapped fluxon is characterized by a minimum of $I_{c}(H)$ at $H \approx 0$.

At $H=0$ the fluxon depinning current $I_{\text {cr }}$ is very small, by

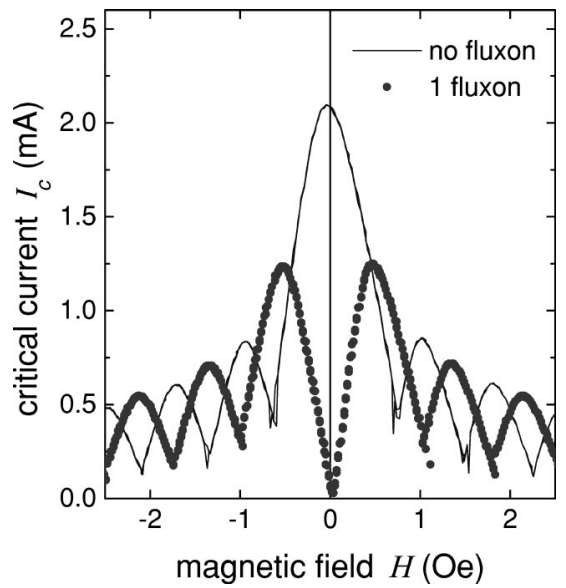

FIG. 2. Critical current dependence on magnetic field measured in the annular junction with no fluxon (solid line) and with one fluxon trapped (points) during cooling down through $T_{c}^{\mathrm{Nb}}$.

a factor of about 300 smaller than the critical current $I_{\mathrm{c}}$ measured for the same junction without the trapped fluxon. This indicates a very high homogeneity of the junction. The linear increase of $I_{\text {cr }}$ at low field $H$ is well described by the theoretical model ${ }^{8}$ based on Eq. (1): the zero-voltage state exists as long as the maximum pinning force due to the fieldinduced potential is larger than the bias current force acting on the fluxon. This is satisfied in the range $|\gamma|<\gamma_{\text {cr }}$ where $^{8}$ $\gamma_{\mathrm{cr}}=h \operatorname{sech}\left(\pi^{2} / l\right)$. In the low-field range, fluxon pinning and retrapping by the magnetic field-induced potential have been recently studied in experiments and analytically in Ref. 12 . The nonlinear dependence of $I_{\mathrm{cr}}(H)$ at high fields with a different number of trapped fluxons has been investigated earlier in detail by Vernik et al. ${ }^{11}$.

The fluxon's $I-V$ characteristics at low magnetic fields and three different temperatures are shown in Fig. 3. As indicated on the plot, different curves correspond to different values of the magnetic field. With increasing $H$ the critical current $I_{\mathrm{cr}}$ increases, and hysteresis appears on the $I-V$ curves. At $I>I_{\mathrm{cr}}$ the fluxon overcomes the pinning potential and starts to move in the junction, which induces dc voltage. If the bias current is decreased, the underdamped fluxon motion continues until the current is low enough for the fluxon to be retrapped by the well. ${ }^{12}$ The $I-V$ characteristics presented in Fig. 3(a) show a clear resonant step at 28-30 $\mu \mathrm{V}$. First, at small $H$, the $I-V$ curve shows a little bump at about $30 \mu \mathrm{V}$ which evolves into a well-pronounced step at larger fields. At fields larger than about $0.15 \mathrm{Oe}$, this step disappears due to the increase of the retrapping current.

The shape of the resonant step strongly depends on temperature. Figures. 3(b) and 3(c) show the $I-V$ characteristics measured at lower temperatures. The asymptotic voltages of the fluxon step and of the field-induced resonant step increase at lower temperatures due to the decrease of the London penetration depth of $\mathrm{Nb}$. At low temperatures, the resonance very clearly shows a backbending, i.e., negative differential resistance in some current range. The backbending is observed starting from the field $H \approx 0.08$ Oe. Decreasing temperature makes this feature sharper, as can be seen from Fig. 3(c). In this plot we can also notice additional small bumps on the $I-V$ curve which appear at lower voltages, at about $22 \mu \mathrm{V}$ and $36 \mu \mathrm{V}$. 

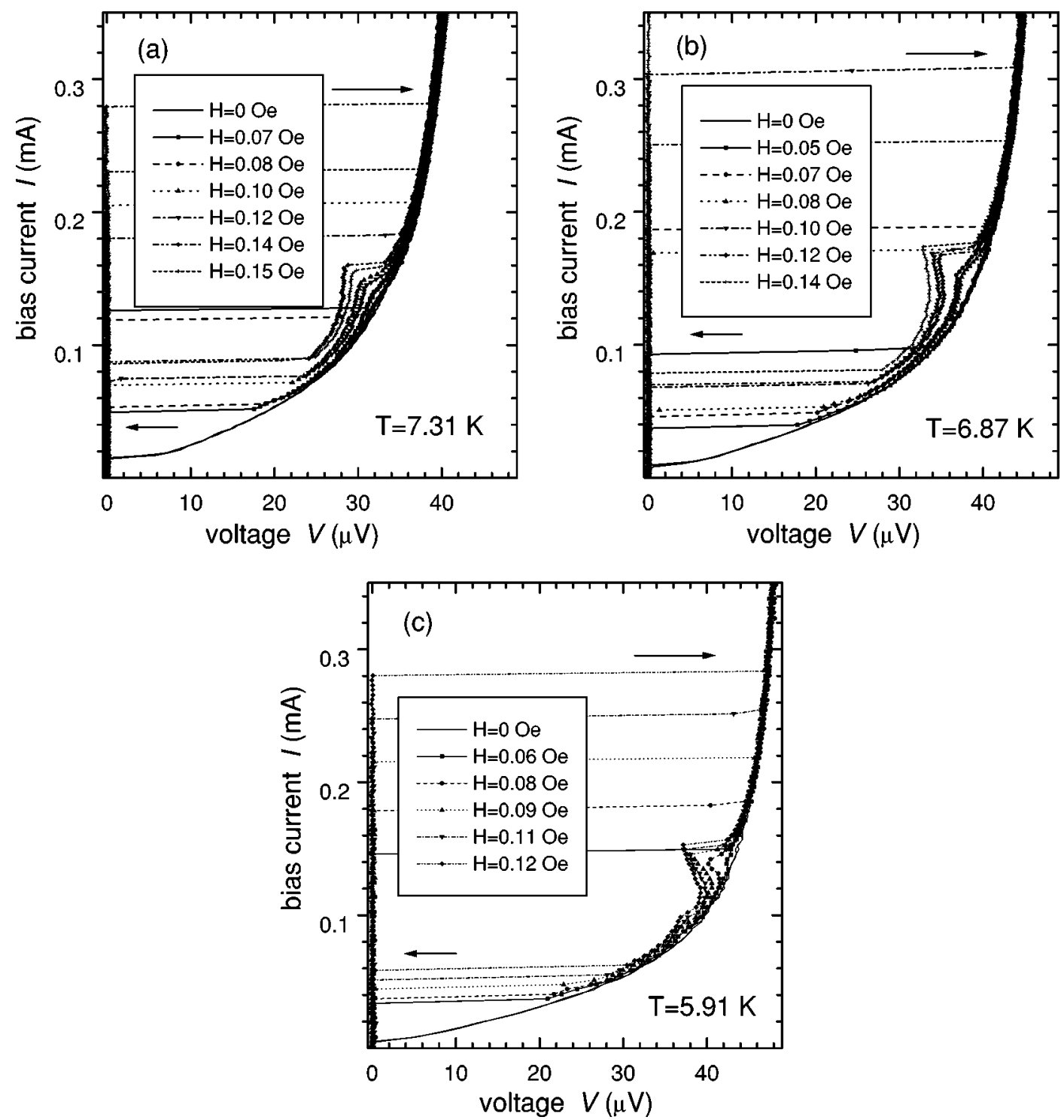

FIG. 3. Current-voltage characteristics of a single fluxon moving in the junction at $T=7.3 \mathrm{~K}$. Different applied magnetic fields $H$ are indicated on the plot. Horizontal arrows show switching directions. (a) $T=7.31 \mathrm{~K}$ with $\alpha \approx 0.052$, (b) $T=6.87 \mathrm{~K}$ with $\alpha \approx 0.043$, and (c) $T=5.91 \mathrm{~K}$ with $\alpha \approx 0.030$.

\section{NUMERICAL SIMULATIONS}

We have calculated the current-voltage curves by numerically integrating Eq. (1). In the simulations, the $I-V$ curve is determined by the dependence of the fluxon velocity $v$ on the bias current $\gamma$. The periodic boundary condition $\varphi(l, t)$ $=2 \pi+\varphi(0, t)$ has been used. The simulation results with the parameters $l=7.8$ and $\alpha=0.05$, close to that in the experiment shown in Figs. 3(b) and 3(c), are presented in Fig. 4. One can see that the simulations show very clearly a rather good qualitative agreement with the experimental data of Fig. 3. The magnetic field $h$ induces a resonance step at the fluxon velocity of about 0.75 . With increasing $h$, this step becomes very pronounced and, finally, at $h=0.7$ it shows the backbending behavior.

The internal dynamics of the junction corresponding to the simulated $\gamma(v)$ characteristics can be learned from the time dependence of the instantaneous Josephson voltage as shown in Fig. 5. Cases (a) and (b) correspond to the stable points on the simulated current-voltage curves at the fieldinduced resonant step (a) and on the main fluxon step (b). One can see that the resonant step at $v \approx 0.75$ is characterized by the background voltage oscillations (plasma waves) with a time period 3 times smaller than the fluxon oscillation period. Thus, at the resonant regime (a) the fluxon strongly interacts with the field-induced potential and a large part of its energy is transferred into the radiation.

In order to investigate the dependence of the resonant features on the junction parameters, we performed numerical simulations for different lengths of the junction. With increasing the junction length $l$, we have found that the main field-induced resonance shifts towards high velocities. In addition, for relatively small damping, other resonant steps appear at lower velocities. Figure 6 presents an example of the $I-V$ curve for a long ring $l=25$ with small damping $\alpha$ $=0.01$ and magnetic field amplitude $h=0.3$. There are three major resonant features at $v \approx 0.81,0.89$, and 0.96 . 


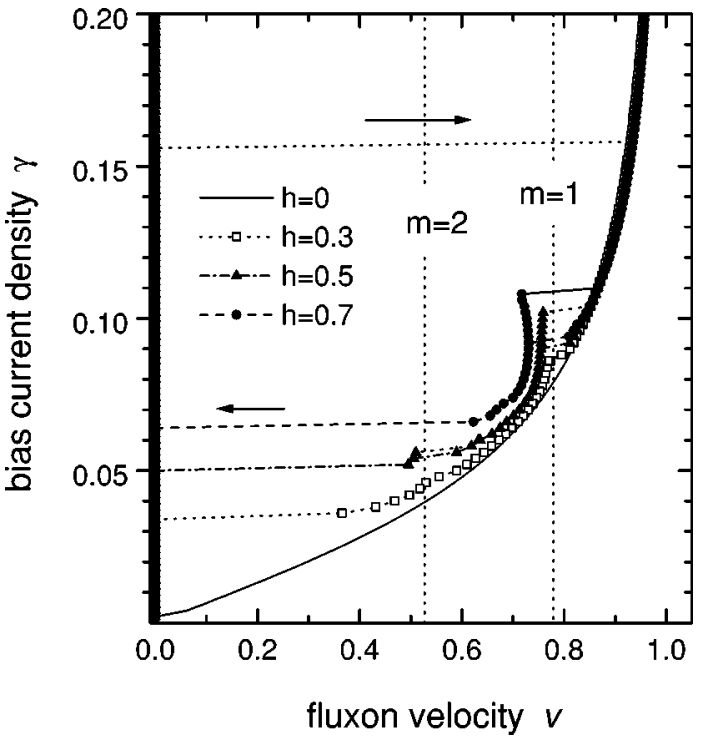

FIG. 4. Numerically simulated current-voltage characteristics of a single fluxon for the junction's parameters $l=7.8, \alpha=0.05$, and $h$ as indicated on the plot. The resonant step associated with the interaction of the fluxon with its radiation is seen at $v \approx 0.75$. The resonance at $h=0.7$ shows clear backbending.

\section{THEORY}

A. Calculation of the resonance frequency: Fluxon interaction with small-amplitude Josephson plasma waves

A simple model for the fluxon resonance due to the Josephson plasma-wave radiation has been put forward in Ref.
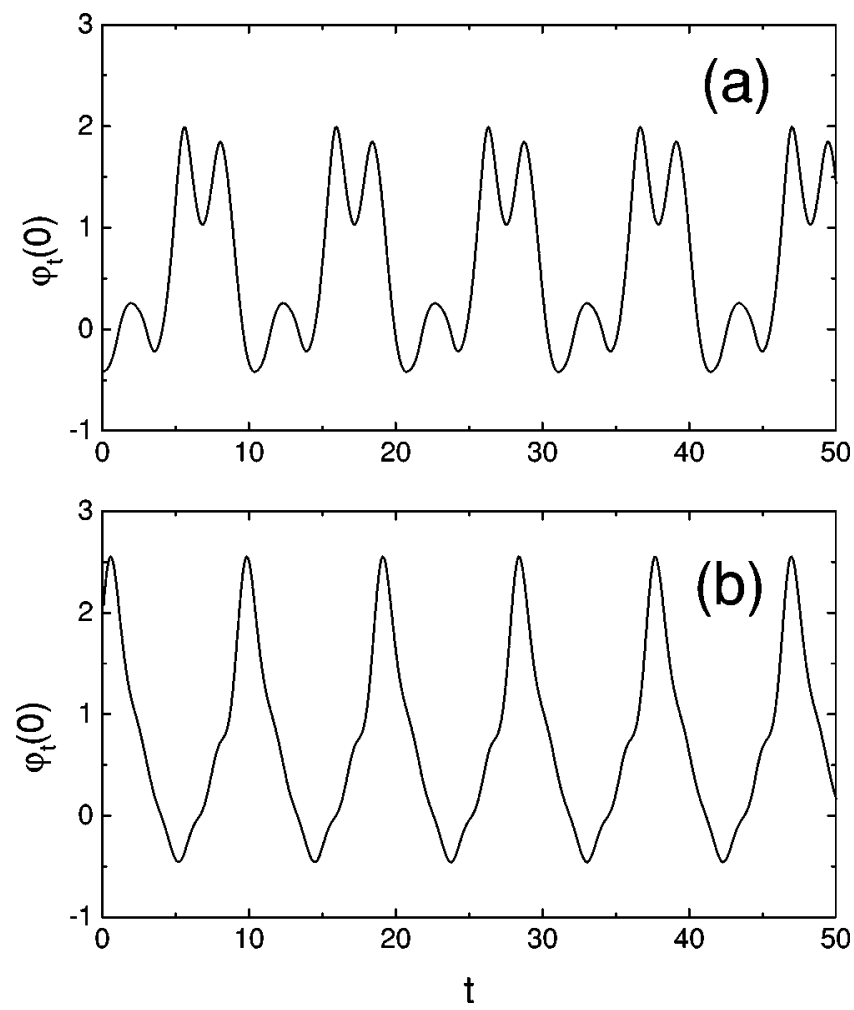

FIG. 5. Voltage oscillations at $x=0$ for two different points of the $\gamma(v)$ curve with $h=0.5$ shown in Fig. 4: (a) at the radiationinduced step $(\gamma=0.09, v=0.75)$ and (b) at the main fluxon step $(\gamma=0.10, v=0.84)$.

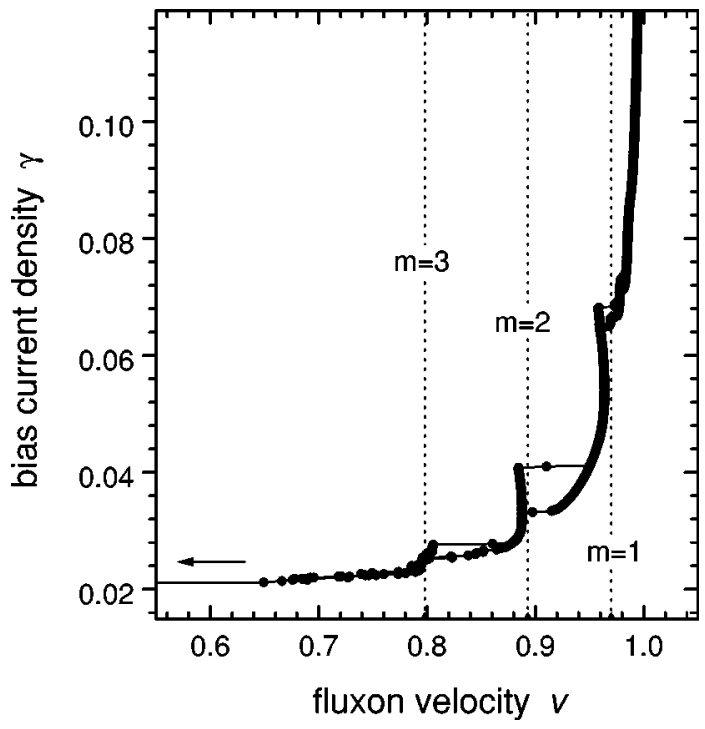

FIG. 6. Numerical simulations of the fluxon in a long ring ( $l$ $=25$ ) with small damping $\alpha=0.01$. Magnetic field amplitude is $h$ $=0.3$. Dashed lines show fluxon velocities corresponding to the thresholds of different harmonics of fluxon radiation.

10. Here we discuss it in more detail in order to explain the main features of the experiments and numerical simulations presented above.

A fluxon moving in an annular junction can be viewed as a particle moving in a periodic potential with a spatial period equal to $l$. Under such conditions, the fluxon is predicted ${ }^{2}$ to emit small-amplitude plasma waves with wave number $k$ and frequency $\omega=\sqrt{1+k^{2}}$ which depend on the period $l$ and the fluxon velocity $v$. According to Mkrtchyan and Schmidt, ${ }^{2}$ the amplitude of the emitted waves is largest near the radiation threshold. At velocities below the threshold the fluxon does not emit radiation because of the Josephson plasma gap in the dispersion relation. The fluxon velocity corresponding to the radiation threshold is given by the following formula: ${ }^{2}$

$$
v_{\text {thr }}=\frac{1}{\sqrt{1+(2 \pi / a)^{2}}},
$$

where $a$ is the period of the potential (in the present case, actually, $a=l$ ).

In a finite system, the radiation should lead to a series of resonances between the fluxon circulation frequency $\omega_{\mathrm{fl}}$ $=2 \pi v / l$ and the frequency of emitted radiation, at $\omega$ $=n \omega_{\mathrm{fl}}$, with $n$ being an integer. These resonances where predicted $^{4,14}$ to induce steps on $I-V$ characteristics at the fluxon velocities

$$
v_{n}=\sqrt{\left(1-\frac{l}{n a}\right)^{2}+\left(\frac{l}{2 \pi n}\right)^{2}} .
$$

Using Eq. (3) with $a=l=7.8$, we obtain $v_{3} \approx 0.785$ to be the closest resonance to the threshold velocity $v_{\text {thr }} \approx 0.779$. This prediction is in good agreement with the experimentally measured (Fig. 3) and numerically calculated (Fig. 4) position of the resonance step. Moreover, the radiation frequency in Fig. 5(a) corresponds to $n=3$, as predicted by this model. Figure 7 shows the resonance velocities $v_{n}$ of the fluxon 


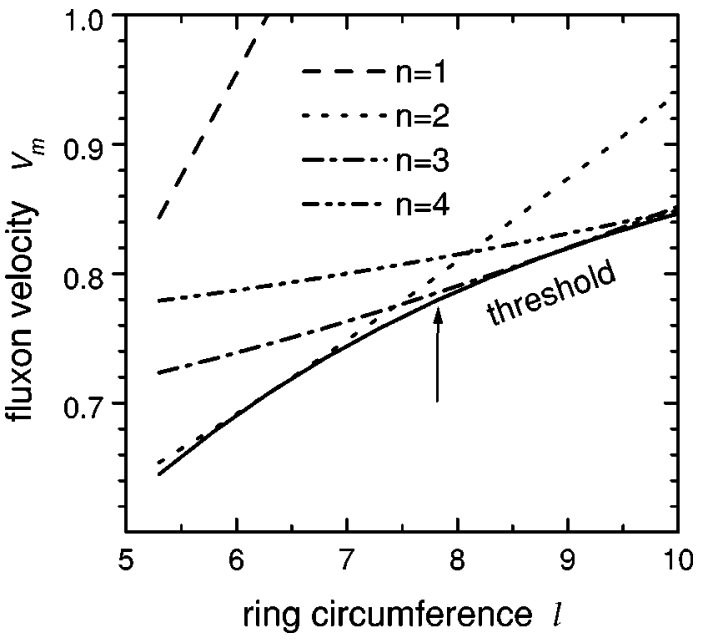

FIG. 7. Dependence of the resonance velocities $v_{n}$ for the first harmonic $(m=1)$ of the fluxon radiation as a function of the junction length. The solid line shows the radiation threshold velocity. The arrow indicates the junction length $l=7.8$ approximately corresponding to the experimentally [Fig. 3(b)] and numerically (Fig. 4) studied cases.

radiation versus the junction length $l$. The solid line shows the radiation threshold velocity (2).

At low damping, the radiation decay time can be very long, and the emitted waves can survive several fluxon circulation periods. This means that, in such a case, high spatial harmonics of the periodic potential can contribute into the dynamics. The formula for the radiation threshold (2) is modified to

$$
v_{\mathrm{thr}}=\frac{1}{\sqrt{1+(2 \pi m / a)^{2}}},
$$

where $m$ is the harmonic number. The dashed lines in Fig. 4 and Fig. 6 show the fluxon velocities corresponding to the thresholds of different harmonics $m$ of the fluxon radiation. One can see that the agreement with numerically calculated resonances is very good.

\section{B. Strong interaction: Analytical approach to explain the backbending $I-V$ curves}

The problem formulated above, i.e., explanation of the conspicuous backbending section on the $I-V$ curve of a fluxon moving in a circular Josephson junction in the external magnetic field, is a challenge for analytical considerations based on the perturbation theory for solitons in the sine-Gordon equation. ${ }^{3}$ As we will see below, a crucially important element that must be added to this well-established technique is a downshift of the junction's plasma frequency under the action of a finite-amplitude radiation field. Of course, it is difficult to develop an absolutely rigorous perturbation theory in the presence of a finite-amplitude background; nevertheless, we will demonstrate below that quite reasonable and not too complicated results can be obtained on the basis of a simple self-consistent approximation.

The model which furnishes quite an accurate description of this system is the perturbed sine-Gordon equation (1). In the absence of perturbations, the fluxon is described by the kink solution to the sine-Gordon equation,

$$
\varphi_{\text {kink }}=4 \tan ^{-1}\left[\exp \left(\frac{\sigma(x-v t)}{\sqrt{1-v^{2}}}\right)\right],
$$

where $v$ is the kink's velocity and $\sigma= \pm 1$ is its polarity.

A well-known result of the perturbation theory ${ }^{2,3}$ is that, for a fluxon moving in a periodic potential (generally speaking, of an arbitrary form) with period $a=2 \pi / q$ (in our case, it is simply equal $l$ ), a strong resonant emission of quasilinear dispersive waves by the fluxon appears when its velocity attains the threshold value

$$
v_{0}=\frac{1}{\sqrt{1+q^{2}}}
$$

which is tantamount to Eq. (2). In this analysis we will assume, as above, that the radiation takes place at some wave number $q_{m}=2 \pi m / a$, which gives a resonance at some velocity very close to the threshold (6). Close to the velocity (6), i.e., at small $\delta v \equiv v-v_{0}$ (which may be both positive and negative), a general expression for the emission power $W$, i.e., the rate at which energy is emitted by the fluxon, was obtained in Ref. 15:

$$
W^{2}=C h^{4} \frac{\sqrt{(\alpha q)^{2}+4\left(1+q^{2}\right)^{2}(\delta v)^{2}}+2\left(1+q^{2}\right) \delta v}{\left[q^{2}(\alpha q)^{2}+4\left(1+q^{2}\right)^{2}(\delta v)^{2}\right]},
$$

where all information about a particular form of the emission-generating perturbation condenses into a single numerical constant $C$; notice that the smallness parameter $h$ of the perturbation (proportional to the external magnetic field) was separated from this constant, to explicitly show at which order of the perturbation theory the result has been obtained.

The resonant character of expression (7) is quite obvious: if one omits $\alpha$, the emission power diverges at $\delta v=0$, while the dissipation prevents this divergence. Nevertheless, at a finite but small $\alpha$ the emission power (7), regarded as a function of $\delta v$, has a sharp maximum around $\delta v=0 .{ }^{15}$

An important step to be done in the present analysis is to take into regard a finite amplitude of the background radiation in an established regime of motion of the fluxon. If the radiation's amplitude is finite but still small enough, one expands the unperturbed sine-Gordon equation in a straightforward way:

$$
\varphi_{t t}-\varphi_{x x}+\varphi-\frac{1}{6} \varphi^{3}=0 .
$$

A solution to Eq. (8) can be looked for, as usual, in the form of an anharmonic expansion

$$
\varphi(x, t)=A \cos (k x-\omega t)+\cdots,
$$

which immediately leads to the (weakly) nonlinear dispersion relation for the finite-amplitude radiation:

$$
\omega^{2}=\omega_{0}^{2}+k^{2}, \quad \omega_{0}^{2} \equiv 1-\frac{1}{8} A^{2}
$$


In this relation, $\omega_{0}^{2}$ is nothing else but an effective plasma frequency (frequency gap) of the junction, with a decreasing correction originating from the finite-amplitude background. Strictly speaking, the finite-amplitude background is subject to a modulational instability. However, for a small background's amplitude this is a very weak effect, which can be additionally suppressed by the dissipation (see below), therefore we ignore it.

The change of the effective plasma frequency will also change the resonant velocity $v_{0}$ as follows [cf. Eq. (6)]:

$$
\tilde{v}_{0}=\frac{\omega_{0}}{\sqrt{\omega_{0}^{2}+q^{2}}} .
$$

Although the difference between $\tilde{v}_{0}$ and $v_{0}$ is small, as we assume the correction $\frac{1}{8} A^{2}$ in Eq. (10) to be small, it may considerably change the effective velocity deviation from the resonant value:

$$
\widetilde{\delta} v \equiv v-\tilde{v}_{0} \approx \delta v+\frac{1}{2} q^{2}\left(1+q^{2}\right)^{-3 / 2}\left(1-\omega_{0}^{2}\right),
$$

where the smallness of $\left(1-\omega_{0}^{2}\right)$ [see Eq. (10)] has been used to expand expression (11).

The next step is to relate the emission power $W$ and the squared amplitude $A^{2}$ of the radiation background. To this end, we consider radiation energy $E$, which, in the linearwave approximation, is

$$
E=\frac{1}{2} \int_{-\infty}^{+\infty}\left(\varphi_{t}^{2}+\varphi_{x}^{2}+\varphi^{2}\right) d x
$$

In the near-resonant case, the emitted waves have small wave numbers. ${ }^{15}$ Therefore, in the first approximation, the gradient (second) term in Eq. (13) may be neglected. Then, substituting the simple wave form (9) into Eq. (13), we easily obtain

$$
E=\frac{1}{2} A^{2} l,
$$

$l$ being, as above, the length of the junction. In the same approximation, it is easy to calculate the rate of dissipation of the energy: $(d E / d t)_{\text {diss }}=-\alpha E$, where $\alpha$ is the dissipative constant in Eq. (1). With regard to this, the balance equation for the energy is

$$
\frac{d E}{d t}=W-\alpha E,
$$

$W$ being the emission power defined above. A stationary solution to Eq. (15) is evident, $E_{0}=W / \alpha$. Finally, equating it to expression (14), we arrive at the following expression for the stationary value of the squared background amplitude in terms of the emission power:

$$
A_{0}^{2}=\frac{2 W}{\alpha l} .
$$

Now, we can obtain a closed self-consistent approximation, inserting expression (16) into Eq. (12), and then substituting the latter expression, instead of $\delta v$, into Eq. (7). To the best of our knowledge, this is the first example of a calculation of radiation effects in the perturbed sine-Gordon model which takes into account the background-induced narrowing of the junction's plasma frequency gap, i.e., actually, the nonlinear character of the emitted radiation.

The form of the resultant equation is radically simplified by the rescalings

$$
\begin{gathered}
W \equiv 8 \pi \alpha^{2} q^{-2} \sqrt{1+q^{2}} P, \\
C h^{4} \equiv(8 \pi)^{2} \alpha^{5}\left(1+q^{2}\right) \mathcal{E}, \\
\delta v=\frac{1}{2} \alpha q\left(1+q^{2}\right)^{-1} \Delta,
\end{gathered}
$$

where $P, \Delta$, and $\mathcal{E}$ are, respectively, the rescaled emission power, velocity deviation, and the perturbation-strength parameter. In terms of this notation, the final equation following from Eqs. (16), (12), and (7) takes a relatively simple form:

$$
P^{2}=\mathcal{E} \frac{\sqrt{1+(\Delta+P)^{2}}+(\Delta+P)}{1+(\Delta+P)^{2}},
$$

where we have made use of the identity $l=2 \pi / q$.

Equation (18) gives a relation between the emission power and the velocity (more accurately, velocity deviation). However, the experimentally observable dynamical characteristic of the Josephson junction is its $I-V$ characteristic, i.e., a relation between the bias current density $\gamma$ and the fluxon's velocity $v$; see Sec. II. To obtain the $I-V$ characteristic, we add to Eq. (18), which was obtained, essentially, from the energy-balance equation for the radiation, an equation of motion for the fluxon itself, which can be easily derived as an energy balance for the fluxon, taking into regard the dissipative and radiative losses: ${ }^{3}$

$$
4 v^{2}\left(1-v^{2}\right)^{-1 / 2}+4 \pi \alpha q^{-2} \sqrt{1+q^{2}} P=\frac{\pi \gamma}{\alpha} v,
$$

where $P$ is the rescaled power defined in Eq. (17). A final link between Eqs. (18) and (19) is the relation $v \equiv v_{0}+\delta v$, $v_{0}$ being defined in Eq. (6), where $\delta v$ should be expressed in terms of $\Delta$ as per Eqs. (17).

Note that we did not specify the value of the numerical coefficient $C$ in the underlying expression (7) for the emission power. Its particular value defines the perturbationstrength parameter $\mathcal{E}$ according to Eq. (17) and, in fact, can be adjusted by changing the parameter $h$ in (1), i.e., the strength of the magnetic field.

In Fig. 8, we display a set of the $I-V$ curves obtained from a numerical solution of the system of two algebraic equations (18) and (19) for different values of $\mathcal{E}$ and fixed values of the junction's length, $l=7.8$, and the dissipative constant, $\alpha$ $=0.05$. Using these parameters, the solutions for (a) $\mathcal{E}=1$, (b) $\mathcal{E}=10$, and (c) $\mathcal{E}=100$ are plotted. The most prominent peculiarity of the family of the $I-V$ curves is quite obvious: if the perturbation-strength parameter $\mathcal{E}$ exceeds a critical value $\mathcal{E}_{\text {cr }} \approx 2.71$ (corresponding to $C \approx 0.0068$ for $h=0.6$ ), there appears the backbending on the $I-V$ curves, i.e., exactly the feature that we seek to explain. In other terms, the backbending can be described as negative differential resistance corresponding to a given $I-V$ curve. 


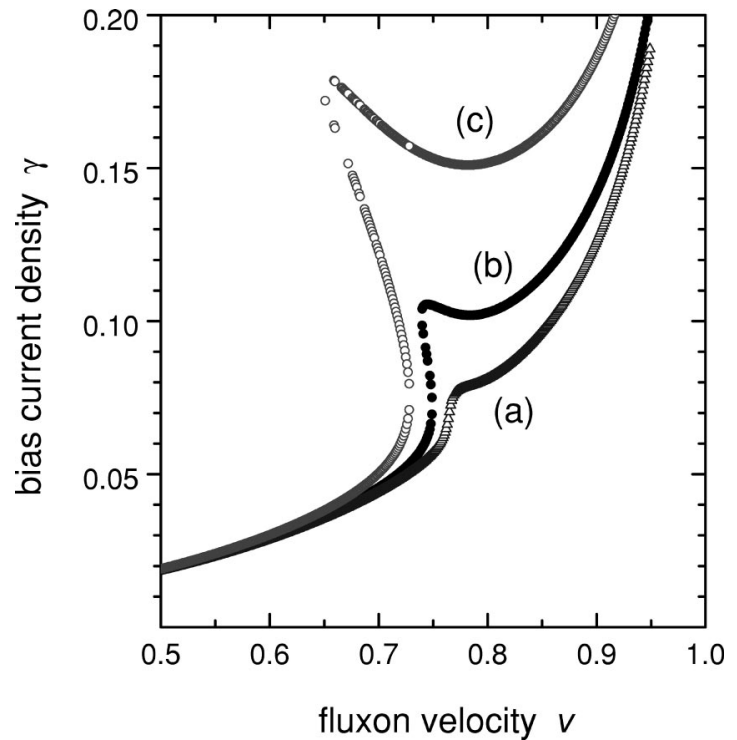

FIG. 8. Analytically derived current-voltage characteristics in the region of the backbending resonance. Magnetic field amplitude is taken as $h=0.6$, dissipation $\alpha=0.05$, and junction's length $l$ $=7.8$. Using these parameters, the solutions for (a) $\mathcal{E}=1$, (b) $\mathcal{E}$ $=10$, and (c) $\mathcal{E}=100$ are plotted.

The increase of the parameter $\mathcal{E}$ means enhancement of the radiative effects and increase of the radiation-background amplitude. This tendency is clearly seen from Figs. 8(a)8(c). Thus, we conclude that a decrease of the effective plasma frequency gap produced by the finite-amplitude radiation background is indeed a crucial fact that allows us to consistently explain the backbending of the $I-V$ curves.

\section{DISCUSSION}

The theory presented in the preceding section demonstrates good overall agreement with experiments and numerically simulated current-voltage characteristics. These facts confirm that an externally applied magnetic field $H$ forms a sinusoidal potential relief for a fluxon in an annular Josephson junction. We have shown that the earlier observed particlelike effects of fluxon pinning and trapping by the potential $^{12}$ are accompanied by the radiation of largeamplitude Josephson plasma waves which are responsible for the novel resonances observed in experiments. In this paper we presented a systematic study of these resonances as a function of the potential height and losses in the junction. We extend here the analogy between the fluxon dynamics in annular junctions and the motion of a particle in a washboard potential. Our study clearly demonstrates that the fluxon behaves as a solitary wave which emits quasilinear waves in a spatially varying potential.

We note that at low temperatures the fluxon radiation step shape becomes more complicated, showing negative differential resistance and chaotic switching between several closely located branches. This behavior is found in detailed study of the resonant step region in Fig. 3(c). Such a complex resonance dynamics was simulated earlier numerically ${ }^{13}$ and was attributed to a strong fluxon-plasma wave interaction leading to an intrinsically chaotic dynamics in the junction. Though the background oscillations were discussed, the authors of Ref. 13 did not suggest any analytical model which can predict the voltage of the resonance or the radiation harmonic content. Based on the model presented in the present paper it can be argued that chaos in this system occurs due to a competition between several resonances lying at close voltages. As an example, for $l=7.8$ the resonances $n=2$ and $n=4$ predicted by Eq. (3) occur at $v_{2} \approx 0.797$ and $v_{4} \approx 0.812$. These values are very close to that of $v_{3}$ and $v_{\text {thr }}$ discussed in Sec. IV A. Thus, the reduction of losses at low temperature can be expected to complicate the dynamics, as was indeed observed in experiment.

\section{ACKNOWLEDGMENTS}

This work was partly supported by Grant No. G0464247.07/95 from the German-Israeli Foundation.
${ }^{1}$ D.W. McLaughlin and A.C. Scott, Phys. Rev. A 18, 1652 (1978).

${ }^{2}$ G.S. Mkrtchyan and V.V. Schmidt, Solid State Commun. 30, 791 (1979).

${ }^{3}$ Yu.S. Kivshar and B.A. Malomed, Rev. Mod. Phys. 61, 763 (1997).

${ }^{4}$ A.A. Golubov and A.V. Ustinov, IEEE Trans. Magn. MAG-23, 781 (1987).

${ }^{5}$ I.L. Serpuchenko and A.V. Ustinov, Pis'ma Zh. Éksp. Teor. Fiz. 46, 435 (1987) [JETP Lett. 46, 549 (1987)].

${ }^{6}$ A. Davidson, B. Dueholm, B. Kryger, and N.F. Pedersen, Phys. Rev. Lett. 55, 2059 (1985).

${ }^{7}$ A.V. Ustinov, T. Doderer, R.P. Huebener, N.F. Pedersen, B. Mayer, and V.A. Oboznov, Phys. Rev. Lett. 69, 1815 (1992).
${ }^{8}$ N. Grønbech-Jensen, P.S. Lomdahl, and M.R. Samuelsen, Phys. Lett. A 154, 14 (1991).

${ }^{9}$ N. Martucciello and R. Monaco, Phys. Rev. B 53, 3471 (1996).

${ }^{10}$ A.V. Ustinov, Pis'ma Zh. Éksp. Teor. Fiz. 64, 178 (1996) [JETP Lett. 64, 191 (1996)].

${ }^{11}$ I.V. Vernik, S. Keil, N. Thyssen, T. Doderer, A.V. Ustinov, H. Kohlstedt, and R.P. Huebener, J. Appl. Phys. 81, 1335 (1997).

${ }^{12}$ A.V. Ustinov, B.A. Malomed, and N. Thyssen, Phys. Lett. A 233, 239 (1997)

${ }^{13}$ N. Grønbech-Jensen, P.S. Lomdahl, and M.R. Samuelsen, Phys. Rev. B 43, 12799 (1991).

${ }^{14}$ A.A. Golubov, I.L. Serpuchenko, and A.V. Ustinov, Zh. Éksp. Teor. Fiz. 94, 297 (1988) [Sov. Phys. JETP 67, 1256 (1988)].

${ }^{15}$ B.A. Malomed, Phys. Lett. A 144, 351 (1990). 